\title{
Ethics Governing Medical Negligence in Clinical Practice
}

\author{
Hizbullah Khan and Neelam Saleem Punjani*
}

Aga Khan University School of Nursing and Midwifery, Karachi, Sindh, Pakistan

*Corresponding author: Neelam Saleem Punjani, MScN (In progress), Aga Khan University School of Nursing and Midwifery, Karachi, Sindh Pakistan, Tel: 713-500-6759; E-mail: neelam.punjani@gmail.com

Rec date: Jun 02, 2014, Acc date: Jul 04, 2014, Pub date: Jul 06, 2014

Copyright: $\odot 2014$ Khan $\mathrm{H}$, et al. This is an open-access article distributed under the terms of the Creative Commons Attribution License, which permits unrestricted use, distribution, and reproduction in any medium, provided the original author and source are credited.

\begin{abstract}
Despite of having appropriate theoretical knowledge, practical skills and competency are necessary for a doctor to perform certain procedure, and if he is not capable of doing the procedure properly and still proceeds for it, this can be counted in malpractice or medical negligence. Any healthcare worker needs to be honest and accountable, and must not violate the ethical boundaries.
\end{abstract}

Keywords: Medical negligence; Ethics; Health care professionals

\section{Introduction}

The principle of beneficence requires doing ethical actions result in obvious and positive benefits to others, while non-maleficence refers to the avoidance of positive harm [1].

This is the story of one of those cases that gave rise to different ethical concerns. A 50 years old male patient was brought to emergency room that had severe chest pain and shortness of breath. After all necessary investigations, he was diagnosed for myocardial infarction and was decided for surgery. Family was consented, his coronary artery bypass grafting was done, and the patient was brought to CICU in critical condition. He was weaned off from ventilator after three days, and he was successfully extubated. Soon after his extubation, he developed breathing difficulty and was going in respiratory failure, so he was planned for elective re-intubation. This time the year one junior resident from anesthesia performed traumatic intubation, and injured the patient's vocal cards badly. Three days after this event, when the doctors were not able to extubate the patient successfully, they realized that the patient has got vocal card paralysis because of the traumatic intubation. Due to vocal card paralysis this patient needed life time tube in his trachea for survival, so his family was told about tracheostomy of the patient. The patient developed pneumonia and multiple other infections due prolong intubation and tracheostomy.

Gradually, this patient got sepsis which led him to multi-organ failure, and irreversible brain injuries. The patient was constantly on ventilator without any physiological improvement. At this time, the family talked to the surgeon that they have sold all their valuables for the treatment, and they have left with nothing more to pay. They were annoyed, and shouting at the doctor that in the initial consent we were told that your patient will be discharged within eight days after surgery, and now it is the 28th post-operative day; our patient is still in miserable condition. The doctor reassured them, they their patient will be alright. Despite the fact that the patient had multi-organ failure and irreversible brain injuries, the doctor continued him on ventilator for few more days. When every medicine was useless to raise his blood pressure, the pacemaker failed pulse generation, and the doctor left with no option, he declared the death to the family.

\section{Medical Negligence}

After critically analyzing the above scenario, it is clear that traumatic intubation was the leading cause for the development of other complications. If the patient did not get vocal card paralysis, he might have gone through successful extubation, and would probably have discharged to home. In addition, neither the family would have suffered from psychological distress, and financial burden, nor would the patient have suffered from unwanted complications for such long duration.

After visualizing the trachea, if this junior doctor had felt difficulty and incompetency, he would have called one of his seniors who are always around, but he didn't do it and proceeded for harmful practice. He didn't even cared to inform anyone after committing the mistake and went away; this is the clear violation of professional values and moral virtue. Although, intubation was aimed for positive benefits of the patient, yet the doctor violated the principle of non-maleficence by his wrong practice.

\section{Kantian Deontology}

Kant believed that an act is morally praiseworthy only if it is done for the motive of obligation and duty, rather than for the self-interests of the person. He added that one must be truthful in every situation even if it carries unwanted short term consequences [2]. In the above scenario, the family members were not aware of the vocal card paralysis of the patient. This information was not shared with them; rather tracheostomy was rationalized to them as the necessitated by their patient's respiratory muscle weakness. There are two purposes of holding this information from the family, one could be the commercial aspect i.e. money making, because if the family knew about this harm initially, they would never have paid the charges. Second purpose is that the doctor might have thought for saving himself and the institute from lawsuits. In the light of ethics, both of these aspects are considered wrong, neither professional values nor the moral values can allow any of those. Deliberately holding the information and lying to the patient or his family is against the Kantian principle of veracity. At the time of complications, multiorgan failure, and brain injury, the family was given false assurance that their patient will come out from the illness and will be discharged. Although, the doctor knew the grave prognosis of the patient, still he 
Page 2 of 2

continued the patient on ventilator against the family will for his own interests. This give rises to two ethical issues, overriding of the family's autonomy, and the violation of deontology.

\section{Futility and Consequentialism}

Futile treatment can be defined as the interventions that are unlikely to be of any benefit to a particular patient in a particular medical condition, and will not achieve the patient's intended goals [3]. Irreversible brain injuries or multi-organ failure are the situations in which treatment can quantitatively delay the death, yet there is no positive effect on quality of life. Such scenarios, in which merely unconsciousness is preserved, are commonly counted under the umbrella of futility. In our case, the patient was purposelessly kept on ventilator for long duration after his multi-organ failure and grave prognosis, which is against the law of bioethics. The doctors are not legally, professionally or ethically required to offer medically futile treatments [4], as it affects the patient and his family both at the same time. On one hand, patient's suffering and troubles are increased by such quantification and futility, on the other hand the financial burden and psychological distress of the family are elevated.

The consequences of futile treatment in our scenario were very obvious as observed ongoing. Once the daughter of the patient told the surgeon that they have sold all their valuables for her father treatment and they are left empty hands. She added that her family is going through financial crisis, if the treatment is of no worth then it needs to be withdrawn, but again she was given the false hopes. She borrowed more money from her relatives to wave off the bills of the hospital, and at the end she got the dead body of her father. Moreover, she was found to visit psychiatric doctor, because she developed psychological symptoms. After analyzing this aspect of the story, it can be assumed that if the doctor was honest enough with this family, he wouldn't have gone for the futile treatment. He would have explained the situation carefully and could have saved the family from financial and psychological crisis.

\section{Utilitarianism and Justice}

The concept of this theory is very broad, but simply it means to promote human welfare by minimizing harms and maximizing benefits [5]. If we take the aspect of futility in the scenario, it is significant that utilitarian approach is violated. The preservation of unconsciousness has maximized harm to the patient and his family, and minimized benefits. On the other hand, this patient has utilized one patient's bed and a ventilator which could have used for saving other patients. CICU is such a place, where usually patients are brought post operatively, kept on ventilator for eight to ten hours, and then shifted to other unit. In this way, the life of five patients can be saved in ten days through one ventilator. If five patients are subjected to futile treatment by the doctors, they will occupy five ventilators for as long as the mechanical support is there. Therefore, other patients who would be better candidates, on the basis of their medical condition and prognosis, for same resources will be automatically deprived of it. This is against utilitarian theory, as it says to maximize benefits for maximum people. Similarly, it is the violation of justice, because of unfair allocation of resources. Justice addresses the equal and fair distribution of health care resources to the clients [6].

\section{Notion of Trust}

It is generally believed that medical professionasls are trusted more than every other professional especially in the Pakistani culture. The reason behind this fact is that, doctors are considered the most honest and knowledgeable people who can truly treat the patients. People blindly believe on each statement of the doctor even if he is wrong. In the above scenario, same situation happened and the family of the patient blindly trusted on every statement of the surgeon. It was only the last day of the patient that his family came to know from someone that their patient has got vocal card paralysis which ended him in such miserable death. They were shocked by this news and hated the doctor like an enemy. The daughter of the patient verbalized that she will no more trust on any of the doctors, and will tell everybody in her community about the story of this specific doctor so that others should not suffer like her. She also gave the story to print media, which posted it a week after the patient's death. As this was a poor family, so they might not have felt themselves capable of taking the matter into court, because of the lawyer charges and probable threats. Due to such scenarios, it is not uncommon that few people can spoil the reputation of whole profession gradually. It is very necessary from ethical and professional point of view that healthcare worker needs to be honest all the times with the patients and families, because once mistrust develops; it is very difficult to regain it, which ultimately affects the quality of care.

\section{Conclusion}

The above discussion concludes that medical professionals need to act in the limits of values and norms of the profession, the law, and the medical ethics. They must not violate the ethical boundaries for their interests to harm the patient instead of benefiting him. As the healthcare workers deal with the lives of patients, they need to be honest all the times even if it brings uneasiness for the short term. Many ambiguous scenarios can come in front in the life of healthcare worker; guidance must be taken from the developed ethical theories and principles, and must be thoroughly discussed for better outcomes. Most of the times, conflicts of one theory or principle with another occurs, so best possible decision must be made to benefit the patient. Along with patients, families' concerns are also very important that need to be taken care of to the extent that does not harm the patients. Lastly, it is necessary to maintain the atmosphere of professional trust through honesty, because it provides basis to mutual decision making and quality care.

\section{References}

1. Agarwal AK (2011) Medical Negligence: Law and Interpretation (No. WP2011-03-03). Indian Institute of Management Ahmedabad, Research and Publication Department.

2. Mulgan T (2007) Ethics: Twelve Lectures on the Philosophy of Moralityby David Wiggins. Philosophical Books 48: 373-376.

3. Burns JP, Truog RD (2007) Futility: A Concept in Evolution. CHEST Journal 132: 1987-1993.

4. Jecker NS \& Pearlman RA (1992) Medical futility: who decides? Archives of internal medicine, 152: 1140-1144.

5. Telfer E (1995) The Elimination of Morality: Reflections on Utilitarianism and Bioethics. Philosophical Books 36: 204-206.

6. Stanley R (1998) Applying the four principles of ethics to continue care. British Journal of Medicine. 